\title{
Simultaneous Mechanical and Topographical Characterization of Alginate Capsules
}

\author{
María Virumbrales-Munoz, Iñaki Ochoa, Luis Fernández \\ Group of Structural Mechanics and Materials Modelling (GEMM) \\ Instituto de Investigación en Ingeniería de Aragón (I3A) \\ Universidad de Zaragoza, Mariano Esquillor s/n, 50018, Zaragoza, Spain. \\ Tel.+34-976762707, e-mail: mavirum@unizar.es
}

\begin{abstract}
We used Atomic Force Microscopy (AFM) in order to characterize alginate microcapsules as for topography and stiffness simultaneously. Thus, we intend to identify those which are theoretically most biocompatible by this technique, reducing the need for animal testing.
\end{abstract}

\section{Introduction}

Microencapsulation is a promising approach for treatment of multiple central nervous system insufficiencies [1] and metabolic disorders, such as diabetes [2]. Therefore, enclosed cells work as customized factories, synthesizing and releasing the desired therapeutic factor in a sustained way [3]. In order to conceal encapsulating materials from the immune system, many groups use poly-L-lysine (PLL). Nonetheless, small changes in this procedure may result in an inadequate binding and strong immune reactions in the host [4]. Nowadays, animal implantation remains the most common way to assess the biocompatibility of the capsules. AFM has previously been used as a powerful tool in order to answer biologically relevant questions [5]. There has been little work on assessing the topography of microcapsules in liquid, given the complexity of topographying in these conditions [6] [7]. In this study, we used AFM in order to characterize surface properties of microcapsules, after different protein treatments, so as to assess their probabilities to induce foreign body response.

\section{Materials and methods}

\section{Encapsulation and protein coating}

Nanobiocel Group (University of the Basque Country) kindly provided the samples for this study. In order to fabricate them, sterile $1.87 \%$ alginate solutions were prepared by dissolving LVG alginate in a $1 \%$ manitol solution and filtering through a 0.50 $\mu \mathrm{M}$ syringe filter. The resulting beads were maintained in agitation for $10 \mathrm{~min}$ in a $\mathrm{CaCl}_{2}$ solution $(55 \mathrm{mM})$ for complete ionic gelation.
Afterwards, different protein coatings were ionically linked to the alginate (A) beads: Poly-L-Lysin (P), genipin $(\mathrm{G})$, genipin-crosslinked double Poly-LLysin (PLL). The resulting combinations were labelled: AP, APG, APP and GDP.

\section{Mechanical and topographical characterization}

For this study, we used QI® mode in JPK Nanowizard 3 coupled with an inverted microscope. This method allows quantitative topography images. In this case Young's Moduli maps were extracted from the force curves used to determine topography images in the first place. To inmobilize the capsules, a nylon mesh was fixed to a petri dish. The dish was first filled with DMEM Medium. After that, 10 $\mu 1$ of the capsule suspension were pipetted on the dish. Then, we approached the cantilever to a capsule and performed force-curve measurements.

\section{Data analysis}

Images and Young's moduli measurements were processed with JPK Data Processing ${ }^{\circledR}$ software. Young's Moduli were calculated by applying the Hertz model to each curve.

\section{Image characterization and analysis}

Images were analyzed with Gwyddion Software for bidimensional statistical parameters. Specifically, characterized sample height, roughness and peakvalley tendency. Furthermore, co-localization curves between topography and Young's Moduli images were calculated using Van Steensel test with Image ${ }^{\circledR}$ software.

\section{Results}

\section{Young's Moduli}

Young's Moduli maps were calculated simultaneously to the topography images (data not shown). During the experiments, exerted force was limited so as to avoid capsule movement. All of the examined capsule types have an apparent Young's 
Modulus in the GPa range. GDP capsules appeared significantly more rigid.

\section{Topography images}

At least 6 images were extracted for each microcapsule type in three different resolutions: 10x10 micron, $3 \times 3$ micron and 500x500 nm (data not shown). Since pore presence is of the essence in this system, $0,5 \times 0,5$ micron regions were investigated to detect smaller pores in the topography, if present. A topography sample can be found in Fig 1B. High vertical resolution was accomplished, and pores consistent with diffusion procedures have been identified.

\section{Colocalization analysis}

Every topographical image was compared to its Young's Moduli image. Whereas AP and APG showed strong co-localization of topographical and Young's Moduli maps, GDP showed almost no colocalization. Interestingly, APP showed an antico-localization pattern. This suggests structural remodelation in the polymer.

\section{Conclusions}

In the presented work, AFM has been applied to characterize alginate microcapsules. Thus, we examined mechanical properties simultaneously to surface topography, in order to establish which surfaces are less likely to induce foreign body reaction when implanted. Thanks to the great precision of this technique, biocompatibility may be eventually assessed without animal testing.

\section{REFERENCIAS}

[1] AEBISCHER, P., GODDARD, M., SIGNORE, A.P., and TIMPSON, R.L. Functional recovery in hemiparkinsonian primates transplanted with polymer-encapsulated PC12 cells. Experimental Neurology. 1994, 126(2), 151-158.

[2] LIM, F., SUM, A.M. Microencapsulated islets as bioartificial endocrine pancreas. Science. 1980, 210(4472), 908910.

[3]. SANTOS, E., PEDRAZ, J.L., HERNANDEZ, R.M., and ORIVE, G. Therapeutic cell encapsulation: ten steps towards clinical translation. Journal of Controlled Release. 2013, 170(1), 1-14.

[4]. VAN STEENSEL et al. Partial colocalization of glucocorticoid and mineralocorticoid receptors in discrete compartments in nuclei of rat hippocampus neurons. Journal of Cell Science, 1996, 109, 787-792.

[5]. DROLLE, E., HANE, F., LEE, B., and LEONENKO, Z. Atomic force microscopy to study molecular mechanisms of amyloid fibril formation and toxicity in Alzheimer's disease. Drug Metabolism Reviews. 2014, 46(2), 207-223.

[6]. ZIMMERMANN, H., HILlGARTNER, M., MANZ B., FEILEN, P., and BRUNNENMEIER F. Fabrication of homogeneously cross-linked, functional alginate microcapsules validated by NMR-, CLSM- and AFM-imaging. Biomaterials. 2003,

24(12),

2083-2096.

[7]. BUNGER, C.M. et al. Biocompatibility and surface structure of chemically modified immunoisolating alginate-PLL capsules. Journal of Biomedical Materials Research Part A. 2003, 67(4), 1219-1227.
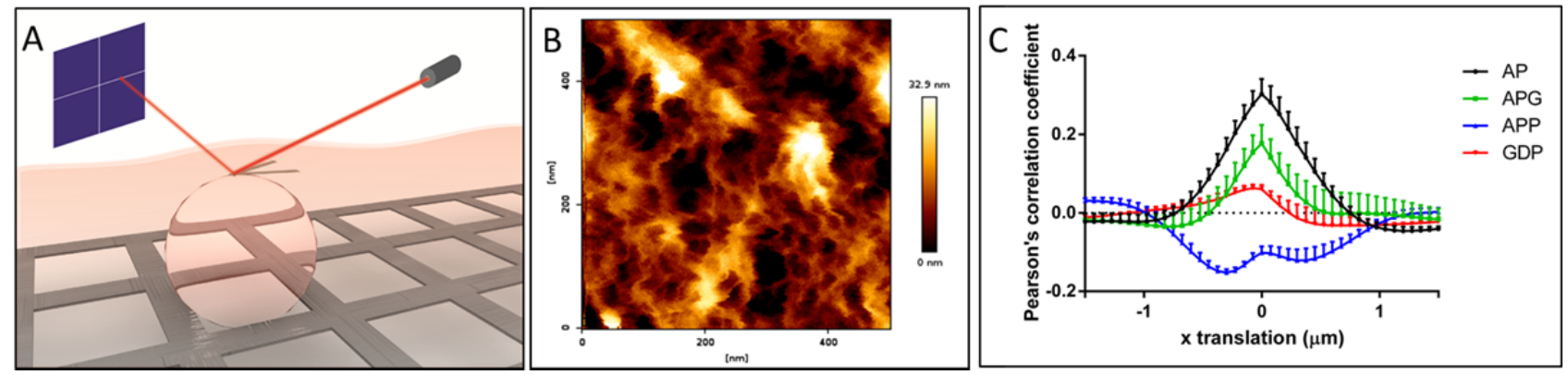

Figure 1: A) Scheme of the AFM setup used for all-the measurements. The measurements always took placewere performed in serum-free culture media, in order to keep the ionic strength for the microcapsules. The nylon mesh was immobilized on an AFM culture dish and the capsules were then added. The cantilever was placed on top of the microcapsule in order to carry out the measurements. B) Sample of topography images taken for the microcapsules. C) Plot showing co-localization between corresponding topography and Young's Moduli images for the different study groups. 